\title{
Electric field effect on magnetism in metallic ultra-thin films
}

\author{
Daichi Chiba * \\ Department of Applied Physics, The University of Tokyo, Tokyo, Japan
}

Recent experimental developments on the electric field effect on magnetism in metallic magnetic materials are reviewed. The change in the electron density at the surface of metallic ultra-thin magnets by the application of an electric field results in modulations of the Curie temperature, magnetic moment, magnetic anisotropy, and domain wall velocity. The study focused on this paper is the electric field effect on the Curie temperature (magnetic phase transition) in Pt/Co ultra-thin film systems. Electric field modifications of the magnetic moment induced by ferromagnetic proximity effects in $\mathrm{Pd}$, which is usually a nonmagnetic element, are also discussed.

Keywords: electric field effect, metallic ultra-thin films, ferromagnetism, phase transition, ferromagnetic proximity effect

\section{OPEN ACCESS}

Edited by:

Atsufumi Hirohata,

University of York, UK

Reviewed by:

Byungchan Han,

Daegu Gyeongbuk Institute of Science and Technology, South Korea

Aurelien Manchon,

King Abdullah University of Science and Technology, Saudi Arabia

*Correspondence: Daichi Chiba dchiba@ap.t.u-tokyo.ac.jp

Specialty section: This article was submitted to Condensed Matter Physics, a section of the journal Frontiers in Physics

Received: 03 July 2015 Accepted: 28 September 2015 Published: 26 October 2015

Citation:

Chiba D (2015) Electric field effect on magnetism in metallic ultra-thin films.

Front. Phys. 3:83

doi: 10.3389/fphy.2015.00083

\section{INTRODUCTION}

Magnets are often needed in modern industry because they have spontaneous magnetization. The direction of the magnetization is utilized as an information bit in magnetic recording media, and the strength of the magnetization is one factor that determines the performance of magnetic devices, e.g., a motor. If the direction or strength of the magnetization can be effectively controlled, the functions and applications of magnetic devices could be expanded, and the dissipation power of these devices will be drastically reduced.

The mechanism for controlling magnetism by the application of an electric field through an insulator is one strong candidate which can fulfill the above expectation $[1,2]$. The electric field control of the Curie temperature [3], coercivity [4], magnetization direction [5, 6], domain wall motion [Yamanouchi_JJAP], and magnetic moment [7] has been experimentally demonstrated using ferromagnetic semiconductors, in which the magnetic property is a function of a carrier concentration that can be modulated by the application of an electric field. These works have defied the previously held belief that the magnetic properties of a material cannot be changed without changing the temperature once the material has been prepared.

Recently, in ferromagnetic metals (FMs), similar results based on the electric field effect have been reported at room temperature [8-11]. Because of the screening effect, the electric field cannot penetrate into the bulk in such metallic systems. Thus, the effect obtained in FMs could be attributed to the change in the electron density at the surface of the material [12, 13]. In particular, at the surface of the $3 d$ transition FMs, the shift of the Fermi level due to the electric field application changes the relative occupation of the $3 d$ orbitals, resulting in the change in the interface magnetic anisotropy $[9,13,14]$. This electrical control of magnetic anisotropy is useful for magnetic recording technology.

Although magnetization switching in magnetic tunnel junctions using spin transfer torque [15-17] is existing technology for writing information in magnetic random access memories, it requires current with very high density; thus, further reduction of unwanted energy dissipation by Joule heating is required. Magnetization switching using the electric field modulation of magnetic 
anisotropy $[6,18-21]$ is expected to reduce the power consumption by more than one or two orders of magnitude because the power required for switching is attributed to charging or discharging of the electrons.

To apply an electric field to the surface of an FM, voltage is applied to a capacitor structure containing an FM electrode. In this structure, the change in the sheet electron density $\Delta n_{\mathrm{S}}$ in the FM electrode by gating corresponds to $C V_{\mathrm{G}} / e$, where $C$ is the capacitance per unit area, $V_{\mathrm{G}}$ is the gate voltage, and $e$ is the electron charge. Therefore, using a device with larger $C$ and applying a larger $V_{\mathrm{G}}$ is the key for realizing a larger $\Delta n_{\mathrm{s}}$. The capacitance is determined by the relative permittivity $k$ and the thickness of the insulator layer $d$ as $C=k \varepsilon_{0} / d$, where $\varepsilon_{0}$ is the vacuum permittivity. To obtain a larger $C$, a higher $k$ and smaller $d$ are required for the dielectric layer.

A conventional approach for obtaining a large $C$ is the adoption of high- $k$ materials (e.g., $\mathrm{Al}_{2} \mathrm{O}_{3}, \mathrm{HfO}_{2}$, or $\mathrm{ZrO}_{2}$ ). Another important consideration is that the capacitor should have a high breakdown field $E_{\mathrm{BD}}\left(=V_{\mathrm{BD}} / d\right.$, where $V_{\mathrm{BD}}$ is the breakdown gate voltage), allowing for the application of a large $V_{\mathrm{G}}$ across the capacitor to obtain a large $\Delta n_{\mathrm{s}}$. Dielectric layers obtained by atomic layer deposition (ALD) are known to have a high $E_{\mathrm{BD}}$ [22]. In particular, if 50-nm-thick $\mathrm{HfO}_{2}$ $(k \sim 20)$ dielectric layer is adopted and $10 \mathrm{~V}$ of $V_{\mathrm{G}}$ (electric field of $0.2 \mathrm{~V} / \mathrm{nm}$ ) is applied, $\Delta n_{\mathrm{s}} \sim 2 \times 10^{13} \mathrm{~cm}^{-2}$. If only the electrons in the topmost mono-layer (ML) of the FM surface can be modulated because of the screening effect, the modulated electron number per FM atom is on the order of $0.01[11]$.

Another way to realize a large $C$ is to use an ionic liquid (or a liquid electrolyte). When a gate voltage is applied between a FM and counter gate electrodes through the ionic liquid, ions are attracted electrostatically to both electrodes; thus, electric double layers (EDLs), which are pairs of sheets of negative and positive charges consisting of the ions in the liquid electrolyte and the induced charges in the surface of the FM electrode (or the gate electrode), respectively, are formed. The EDL capacitor has a very large $C$ because the gap of the two charged sheets, which corresponds to $d$, is $\sim 1 \mathrm{~nm}$ [23]; thus, a large $\Delta n_{s}$ is realized. In this case, the modulated electron number per FM atom can reach the order of 0.1 by the application of a few volts of $V_{\mathrm{G}}$.

The change in the electron density at the FM surface is a reasonable explanation for the electric field control of magnetism. As another candidate, the electric field modulation of the uniaxial perpendicular magnetic anisotropy (PMA) caused by the Rashba effect at the interface between the two different materials, e.g., a FM metal and nonmagnetic metal or insulator, has been theoretically proposed [24]. Furthermore, the voltage-driven oxidation-reduction reaction or $\mathrm{O}^{2-}$ migration in the $\mathrm{FM}$ /oxide bilayer is known to be another source for altering the magnetic properties of a FM $[25,26]$; however, this effect is much slower than the electron charging effect.

In this paper, recent experimental advances on the electric field control of the ferromagnetic phase transition in $\mathrm{Pt} / \mathrm{Co}$ systems are reviewed. In addition, electric field modifications of the magnetic moment in ferromagnetic $\mathrm{Pd}$, which is usually a nonmagnetic element, deposited on $\mathrm{Pt} / \mathrm{Co}$ system are also discussed.

\section{ELECTRIC FIELD CONTROL OF THE MAGNETIC PHASE TRANSITION}

The author found that not only the magnetic anisotropy but also the ferromagnetism itself is electrically switchable around room temperature in a perpendicularly magnetized $\sim 2$ ML of Co ultrathin film deposited on a Pt underlayer. The layer structure used in the experiment was $\operatorname{Pt}(\sim 1 \mathrm{~nm}) / \mathrm{Co}(0.4 \mathrm{~nm}) / \mathrm{MgO}(2.0 \mathrm{~nm})$ from the bottom side. These layers were deposited on an intrinsic Si substrate by dc or rf-sputtering. The Pt layer was confirmed to have a fcc(111) texture by scanning transmission electron microscopy (STEM) (see Supplementary Online Material of Koyama et al. [27]).

The first experiment was performed using a solid-state capacitor structure with a 50-nm-thick $\mathrm{ALD}-\mathrm{HfO}_{2}$ dielectric insulator layer [11]. In this experiment, the anomalous Hall effect was used to detect the magnetization state; thus, the $\mathrm{HfO}_{2}$ insulator and the gate electrode were formed on top of the Hallbar-mesa structure made of Pt/Co layers. Here, the anomalous Hall resistance $R_{\text {Hall }}$ is proportional to the perpendicular component of the magnetization. An optical microscopy image is shown in the inset of Figure 1A. A clear difference was observed in the magnetization curves detected by the anomalous Hall effect for positive and negative $V_{G}( \pm 10 \mathrm{~V})$ applications, as shown in Figure 1A. (Note that positive $V_{\mathrm{G}}$ is defined as the direction of increasing electron density at the Co surface). When the positive $V_{\mathrm{G}}$ was applied, a clear rectangular hysteresis loop was observed, whereas a linear response was obtained under the application of negative $V_{\mathrm{G}}$. This $V_{\mathrm{G}}$-dependent change in the magnetization curve was observed in a reversible way. From the detailed measurements, e.g., temperature dependence of magnetic susceptibility, this dramatic effect was not attributed to the switching of the magnetic easy axis from the perpendicular to the in-plane direction. This effect was attributed to the phase transition from the ferromagnetic to paramagnetic state. From the Arrott-plot [28] (or the Arrott-Norkes plot [29]), the temperature $T$ dependence of the spontaneous $R_{\text {Hall }}\left(R_{\text {Hall }}^{\mathrm{s}}\right)$ for the application of positive and negative $V_{\mathrm{G}}$, which is proportional to the spontaneous magnetization, was obtained (see Figure 1B). The Curie temperature $T_{C}$ between them was determined to be $\sim 12 \mathrm{~K}$. Because of the two dimensionality of the Co film, $T_{\mathrm{C}}$ in this sample was around room temperature [30].

Ionic-liquid gating was also adopted for modulating the magnetic properties of the similar $\mathrm{Pt} / \mathrm{Co}$ sample [31]. In this experiment, a direct magnetization measurement using a superconducting quantum interference (SQUID) magnetometer was used to detect the magnetization under gating. A polymer film containing the ionic liquid $\left(\mathrm{EMI}^{+}-\mathrm{TFSI}^{-}\right)$was placed on the sample, and a $\mathrm{Pt}$ foil was placed on top to serve as a gate electrode (see the inset of Figure 1C). This film-shaped ionic liquid simplifies the measurement in the SQUID chamber. By applying $V_{\mathrm{G}}$ between the Co layer and the gate electrode, 

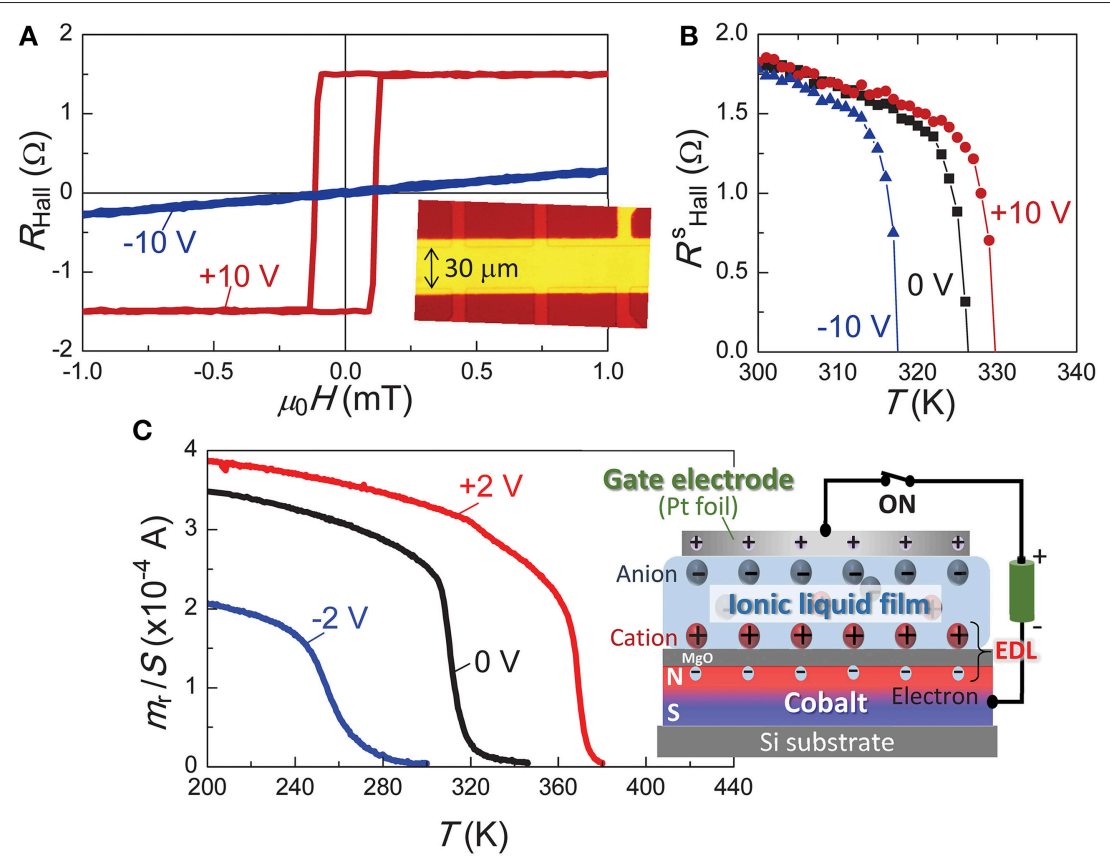

FIGURE 1 | Electric field effect on ferromagnetism in the Pt/Co system. (A) Magnetization curves detected in the Hall resistance $R_{\mathrm{Hall}}$ for $V_{\mathrm{G}}=-10$ and $10 \mathrm{~V}$ at $321 \mathrm{~K}$. The inset shows the top view of the Hall bar structure with the gate electrode on top (yellow region) taken by an optical microscope. Solid-state $\mathrm{HfO}_{2}$ was used as a gate insulator in this device. (B) Temperature $T$ dependence of the spontaneous Hall resistance $R_{\text {Hall }}^{S}$ which is proportional to the spontaneous magnetization obtained with the device in (A). The Curie temperature increases (decreases) with positive (negative) $V_{\mathrm{G}}$. (C) The $T$ dependence of the remanent magnetic moment per unit area $\left(m_{r} / S\right)$ for $V_{G}=+2,0$, and $-2 \mathrm{~V}$. The inset shows the cross-sectional image of the device structure. An ionic liquid was used to gate the sample.

the EDL is formed through the $\mathrm{MgO}$ capping layer, and the electron density at the Co surface can be modulated. Figure 1C shows the $T$ dependence of the remanent magnetic moment per unit area $\left(m_{\mathrm{r}} / S\right)$ for three different $V_{\mathrm{G}}$ values $\left(V_{\mathrm{G}}=+2,0\right.$, and $-2 \mathrm{~V})$. In this device, a giant change in $T_{\mathrm{C}}$ up to $\sim 100$ $\mathrm{K}$ around room temperature was achieved. This is believed to be the result of the larger change in the electron density in the EDL capacitor compared to that in the $\mathrm{HfO}_{2}$ capacitor structure.

In both devices (the ALD- $\mathrm{HfO}_{2}$ and the ionic-liquid-EDL capacitors), the direction of the change in $T_{\mathrm{C}}$ was the same: positive (negative) $V_{\mathrm{G}}$, i.e., the increase (decrease) in the electron density, resulted in higher (lower) $T_{\mathrm{C}}$. Thus, the $T_{\mathrm{C}}$ had a positive slope with respect to the electron density. According to previous reports or ab-initio calculations for a bulk CoNi alloy [32], an increase in the $\mathrm{Ni}$ composition, i.e., the decrease in the electron number per atom, results in the reduction of $T_{\mathrm{C}}$, which is similar to the case of the Slater-Pauling curve (atomic magnetic moment with respect to the electron number for $3 d$ transition metal alloys). The direction of the change in $T_{\mathrm{C}}$ under gating opposed the Salter-Pauling type behavior (negative slope). Recently, the electric field effect on $T_{\mathrm{C}}$ for a similar Pt/Co structure was calculated by ab-initio calculations via Monte Carlo simulations that consider the magnetic anisotropy, and the same direction of the $T_{\mathrm{C}}$ change with the gating experiment was reported [33]. In the paper, it has been pointed out that, because the $s p$ electrons predominantly contribute to the screening of the electric field, the change in the electron number for $d$ electrons opposes that in the total electrons, i.e., the sign of the slope of $T_{\mathrm{C}}$ with respect to the number of $d$ electrons is the same with the Slater-Pauling curve. This can be one reasonable explanation for the experiment. Another possibility is the oxidation-reduction reaction by the application of the electric field $[25,26]$. The negative $V_{\mathrm{G}}$ is in the direction of the oxidation reaction, which should reduce $T_{\mathrm{C}}$ and the magnetic moment of the Co layer. Moreover, the reduction reaction may increase $T_{\mathrm{C}}$ if the Co layer was slightly oxidized after deposition. The evaluation of these effects will be considered in future study.

\section{ELECTRIC FIELD CONTROL OF MAGNETIC MOMENT IN Pd}

The results shown in the previous section show that ferromagnetism is switchable in ferromagnets using an electric field. It is natural to consider whether the electric field can make non-magnets ferromagnetic. Moreover, if one electron is removed from a $\mathrm{Cu}$ atom by an electric field, it is not known if $\mathrm{Cu}$ will show ferromagnetism similar to $\mathrm{Ni}$. This is an extreme example, and it may be impossible to remove one electron from a metallic atom using an electric field. However, this concept may be realized with metals that are similar to ferromagnets. Among these metals, Pd is a nonmagnetic metal that nearly satisfies the Stoner criterion [34-37]. 

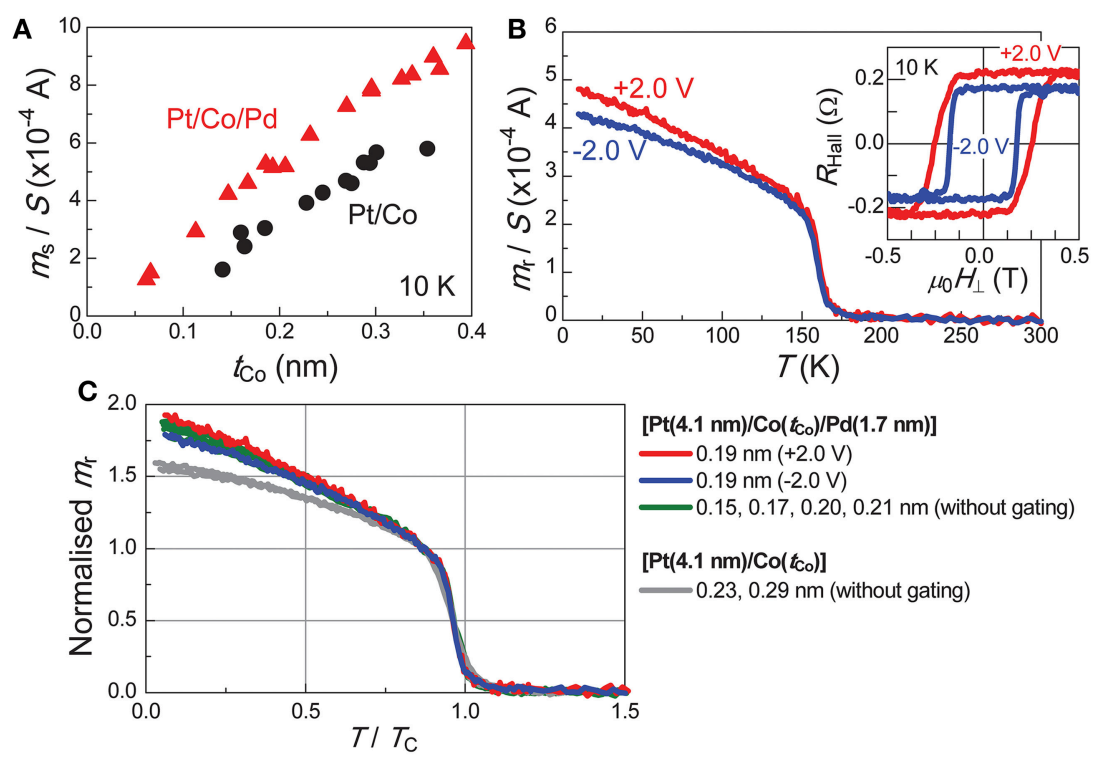

$\left[\mathrm{Pt}(4.1 \mathrm{~nm}) / \mathrm{Co}\left(t_{\mathrm{C}_{0}}\right) / \mathrm{Pd}(1.7 \mathrm{~nm})\right]$

$0.19 \mathrm{~nm}(+2.0 \mathrm{~V})$

$0.19 \mathrm{~nm}(-2.0 \mathrm{~V})$

$0.15,0.17,0.20,0.21 \mathrm{~nm}$ (without gating)

$\left[\mathrm{Pt}(4.1 \mathrm{~nm}) / \mathrm{Co}\left(t_{\left.\mathrm{C}_{0}\right)}\right)\right]$

$0.23,0.29 \mathrm{~nm}$ (without gating)

FIGURE 2 | Electric field effect on the induced magnetic moment in Pd. (A) The Co thickness ${ }_{t}$ Co dependence of the saturation magnetic moment per unit area $\left(m_{\mathrm{S}} / S\right)$ at $10 \mathrm{~K}$ for the $\mathrm{Pt}(4.1 \mathrm{~nm}) / \mathrm{Co}\left(t_{\mathrm{Co}}\right)$ and $\mathrm{Pt}(4.1 \mathrm{~nm}) / \mathrm{Co}\left(t_{\mathrm{Co}_{0}}\right) / \mathrm{Pd}(1.7 \mathrm{~nm})$ samples. (B) The $T$ dependence of the remanent magnetic moment per unit area $\left(m_{r} / S\right)$ for the $\mathrm{Pt}(4.1 \mathrm{~nm}) / \mathrm{Co}(0.19 \mathrm{~nm}) / \mathrm{Pd}(1.7 \mathrm{~nm})$ sample under positive $(+2 \mathrm{~V})$ and negative $(-2 \mathrm{~V}) V_{\mathrm{G}}$. The inset shows the magnetic hysteresis loops for both $V_{\mathrm{G}}$ at $10 \mathrm{~K}$. For the hysteresis measurement, the anomalous Hall effect was used. (C) The $T / T_{\mathrm{C}}$ dependence of the normalized $m_{\mathrm{r}}$ for the $\mathrm{Pt} / \mathrm{Co}$ and $\mathrm{Pt} / \mathrm{Co} / \mathrm{Pd}$ samples with various $t_{\mathrm{Co}}$. The $m_{\mathrm{r}}$ was normalized at $T / T_{\mathrm{C}}=0.87$. All data points without gating were sandwiched by the positively and negatively gated data points, indicating that the induced magnetic moment at the surface of the Pd layer was modulated by the application of an electric field.

Ab-initio calculations have shown that the peak of the density of states of bulk nonmagnetic Pd is located at an energy near the Fermi level [34], suggesting that an applied electric field may affect the magnetic state in Pd [37]. Although the modulation of paramagnetic properties was observed in $\mathrm{Pt}$ thin films using the EDL capacitor [38], electric-field-induced ferromagnetism has not been reported in nonmagnetic metals. Thus, as a first step, the tenability of the induced magnetic moment in $\mathrm{Pd}$ by the ferromagnetic proximity effect has been investigated [39].

A magnetic moment is induced by the ferromagnetic proximity effect in a Pd or Pt layer deposited on a ferromagnetic metal layer [40-45]. Figure 2A shows the Co thickness $t_{\text {Co }}$ dependence of the saturation magnetic moment per unit area $\left(m_{\mathrm{s}} / S\right)$ at $10 \mathrm{~K}$ for the $\mathrm{Pt}(4.1 \mathrm{~nm}) / \mathrm{Co}\left(t_{\mathrm{Co}}\right)$ and $\mathrm{Pt}(4.1 \mathrm{~nm}) / \mathrm{Co}\left(t_{\mathrm{Co}}\right) / \mathrm{Pd}(1.7 \mathrm{~nm})$ samples. The $\mathrm{Pd}$ and Pt layers were confirmed to have an fcc [?] texture using X-ray diffraction. Both samples were capped by 2-nm-thick MgO layer. For both series, $m_{\mathrm{s}} / S$ increases with $t_{\mathrm{Co}}$. However, the samples with the $\mathrm{Pd}$ layer showed larger $m_{\mathrm{s}} / S$ than the Pt/Co samples, indicating that a magnetic moment was induced in the Pd layer. Assuming that the magnetic moment is uniformly induced in the entire Pd layer, the induced magnetic moment per Pd atom is calculated to be $\sim 0.1 \mu_{\mathrm{B}}$ ( $\mu_{\mathrm{B}}$ is the Bohr magneton), the order of which is in good agreement with previous studies [40, 42, 43]. The Pd thickness dependence of the magnetic moment (not shown) indicated that the magnetic moment was induced up to $2 \mathrm{~nm}$ from the $\mathrm{Pd} / \mathrm{Co}$ interface. Thus, the Pd surface of the samples shown in Figure 2A is expected to be ferromagnetic. Note that no superparamagnetic behavior was observed in $\mathrm{Pt} / \mathrm{Co} / \mathrm{Pd}$ samples even when the thickness of the Co layer was about half monolayer $(\sim 0.1 \mathrm{~nm})$. In addition, the decrease in $T_{\mathrm{C}}$ and perpendicular magnetic anisotropy were confirmed after annealing of several $\mathrm{Pt} / \mathrm{Co} / \mathrm{Pd}$ samples at 200 or $300^{\circ} \mathrm{C}$, suggesting that there was much less intermixing of $\mathrm{Pd}$ or $\mathrm{Pt}$ with Co before annealing.

Figure 2B shows the $T$ dependence of the remanent magnetic moment per unit area $\left(m_{\mathrm{r}} / S\right)$ for one of the $\mathrm{Pt} / \mathrm{Co} / \mathrm{Pd}$ samples with $t_{\mathrm{Co}}=0.19 \mathrm{~nm}$ under positive and negative $V_{\mathrm{G}}$. Though $T_{\mathrm{C}}$ was not visibly changed, a clear difference was observed in $m_{\mathrm{r}} / S$, and the difference increased with decreasing temperature. The squareness ratio was almost one for both $V_{\mathrm{G}}$ values at low $T$ as shown in the inset; thus, this change was attributed to the change in the induced magnetic moment at the surface of the Pd layer. Two samples with different $t_{\mathrm{Co}}$ values were investigated. The modulated atomic magnetic moment for $V_{\mathrm{G}}=$ $1 \mathrm{~V}$ was calculated to be $0.05-0.08 \mu_{\mathrm{B}}$, and the modulated electron number per Pd atom was estimated to be $0.03-0.05$ from the measured capacitance of each device.

Figure 2C shows the $T / T_{\mathrm{C}}$ dependence of the normalized $m_{\mathrm{r}}$ for $\mathrm{Pt} / \mathrm{Co}$ and $\mathrm{Pt} / \mathrm{Co} / \mathrm{Pd}$ samples with various $t_{\mathrm{Co}}$. The $m_{\mathrm{r}}$ was normalized at $T / T_{C}=0.87$. In general, a larger increase in the magnetic moment was observed in the $\mathrm{Pt} / \mathrm{Co} / \mathrm{Pd}$ samples as $T$ decreased. This behavior is probably due to the larger magnetic susceptibility of the Pd layer at lower $T[43,45]$. All data points for samples without gating were sandwiched by the data points from the positively and negatively gated ones in $\mathrm{Pt} / \mathrm{Co} / \mathrm{Pd}$ samples. This is one example showing that the induced 
magnetic moment at the surface of the Pd layer was modulated by the application of the electric field. Inducing ferromagnetism in non-magnetic materials is the next challenge.

\section{CONCLUSIONS}

Many experimental results reveal that various magnetic properties are tunable using an electrical gating. Although control of the phase transition and the magnetic moment was emphasized in this paper, the speed of the magnetic domain wall is also tunable using an electrical gating [46-48]. Electricfield-induced magnetization switching $[20,21]$ based on the control of the magnetic anisotropy $[5,9,10]$ should significantly impact future magnetic recording applications. These facts mean that use of the electric field effect has already been established in magnetic materials as well as semiconductors. The electrical

\section{REFERENCES}

1. Ohno H. A window on the future spintronics. Nat Mater (2010) 9:952-4. doi: $10.1038 /$ nmat2913

2. Matsukura F, Tokura Y, Ohno H. Control of magnetism by electric fields. Nat Nanotechnol. (2015) 10:209-20. doi: 10.1038/nnano.2015.22

3. Ohno H, Chiba D, Matsukura F, Omiya T, Abe E, Dietl T, et al. Electric-field control of ferromagnetism. Nature (2000) 408:944-6. doi: 10.1038/35050040

4. Chiba D, Yamanouchi M, Matsukura F, Ohno H. Electrical manipulation of magnetization reversal in a ferromagnetic semiconductor. Science (2003) 301:943-5. doi: 10.1126/science.1086608

5. Chiba D, Sawicki M, Nishitani Y, Nakatani Y, Matsukura F, Ohno H. Magnetization vector manipulation by electric fields. Nature (2008) 455:5158. doi: $10.1038 /$ nature 07318

6. Chiba D, Ono T, Matsukura F, Ohno H. Electric field control of thermal stability and magnetization switching in (Ga, Mn)As. Appl Phys Lett. (2013) 103:142418. doi: 10.1063/1.4821778

7. Sawicki M, Chiba D, Korbecka A, Nishitani Y, Majewski AJ, Matsukura F, et al. Experimental probing of the interplay between ferromagnetism and localization in (Ga, Mn)As. Nat Phys. (2010) 6:22-5. doi: 10.1038/ nphys 1455

8. Weisheit M, Fähler S, Marty A, Souche Y, Poinsignon C, Givord D. Electric field-induced modification of magnetism in thin-film ferromagnets. Science (2007) 315:349-51. doi: 10.1126/science.1136629

9. Maruyama T, Shiota Y, Nozaki T, Ohta K, Toda N, Mizuguchi M, et al. Large voltage-induced magnetic anisotropy change in a few atomic layers of iron. Nat Nanotech. (2009) 4:158-61. doi: 10.1038/nnano. 2008.406

10. Endo M, Chiba D, Shimotani H, Matsukura F, Iwasa Y, Ohno H. Electric double layer transistor with a (Ga, Mn)As channel. Appl Phys Lett. (2010) 96:022515. doi: 10.1063/1.3277146

11. Chiba D, Fukami S, Shimamura K, Ishiwata N, Kobayashi K, Ono T. Electrical control of the ferromagnetic phase transition in cobalt at room temperature. Nat Mater (2011) 10:853-6. doi: 10.1038/nmat3130

12. Duan CG, Velev JP, Sabirianov RF, Zhu Z, Chu J, Jaswal SS, et al. Surface magnetoelectric effect in ferromagnetic metal films. Phys Rev Lett. (2008) 101:137201. doi: 10.1103/PhysRevLett.101.137201

13. Nakamura K, Shimabukuro R, Fujiwara Y, Akiyama T, Ito T, Freeman AJ. Giant modification of the magnetocrystalline anisotropy in transition-metal monolayers by an external electric field. Phys Rev Lett. (2009) 102:187201. doi: 10.1103/PhysRevLett.102.187201

14. Nakamura K, Akiyama T, Ito T, Weinert M, Freeman AJ. Role of an interfacial $\mathrm{FeO}$ layer in the electric-field-driven switching of magnetocrystalline anisotropy at the Fe/MgO interface. Phys Rev B (2010) 81:220409(R). doi: 10.1103/PhysRevB.81.220409 control window of the magnetic properties is expected to continue to increase.

\section{ACKNOWLEDGMENTS}

The author thanks A. Obinata, Y. Hibino, D. Hayakawa, T. Koyama, K. Shimamura, H. Kakizakai, K. Yamada, M. Kawaguchi, K. Kobayashi, T. Ono, K. Miwa, S. Ono, S. Fukami, N. Ishiwata, A. Hirohata, B. Kurebanjiang, V. K. Lazarov, and A. Tsukazaki for their help. This work was partly supported by the PRESTO program of JST, a Grant-in-Aid for Scientific Research (S) (25220604), a Grant-in-Aid for Challenging Exploratory Research (15K13359), and a Grant-in-Aid for Specially Promoted Research (15H05702) from JSPS. A portion of this work was performed using the facilities of the Cryogenic Research Centre of the University of Tokyo.
15. Slonczewski JS. Current-driven excitation of magnetic multilayers. J Magn Magn Mater (1996) 159:L1-7. doi: 10.1016/0304-8853(96)00062-5

16. Berger L. Emission of spin waves by a magnetic multilayer traversed by a current. Phys Rev B (1996) 54:9353-8. doi: 10.1103/PhysRevB.54.9353

17. Brataas A, Kent AD, Ohno H. Current-induced torques in magnetic materials. Nat Mater (2012) 11:372-81. doi: 10.1038/nmat3311

18. Stöhr J, Siegmann HC, Kashuna A, Gamble SJ. Magnetization switching without charge or spin currents. Appl Phys Lett. (2009) 94:072504. doi: $10.1063 / 1.3081421$

19. Chiba D, Nakatani Y, Matsukura F, Ohno H. Simulation of magnetization switching by electric-field manipulation of magnetic anisotropy. Appl Phys Lett. (2010) 96:192506. doi: 10.1063/1.3428959

20. Shiota Y, Nozaki T, Bonell F, Murakami S, Shinjo T, Suzuki Y. Induction of coherent magnetization switching in a few atomic layers of FeCo using voltage pulses. Nat Mater (2012) 11:39-43. doi: 10.1038/nmat3172

21. Kanai S, Yamanouchi M, Ikeda S, Nakatani Y, Matsukura F, Ohno H. Electric field-induced magnetization reversal in a perpendicular-anisotropy $\mathrm{CoFeB}$ MgO magnetic tunnel junction. Appl Phys Lett. (2012) 101:122403. doi: $10.1063 / 1.4753816$

22. Biercuk MJ, Monsma DJ, Marcus CM, Becker JS, Gordon RG. A Lowtemperature atomic-layer-deposition lift-off method for microelectronic and nanoelectronic applications. Appl Phys Lett. (2003) 83:2405-7. doi: $10.1063 / 1.1612904$

23. Yamamoto R, Morisaki H, Sakata O, Shimotani H, Yuan H, Iwasa Y, et al. External electric field dependence of the structure of the electric double layer at an ionic liquid/Au interface. Appl Phys Lett. (2012) 101:053122. doi: $10.1063 / 1.4742920$

24. Barnes SE, Ieda J, Maekawa S. Rashba spin-orbit anisotropy and the electric field control of magnetism. Sci Rep. (2014) 4:4105. doi: 10.1038/srep04105

25. Bonell F, Takahashi YT, Lam DD, Yoshida S, Shiota Y, Miwa S, et al. Reversible change in the oxidation state and magnetic circular dichroism of Fe driven by an electric field at the $\mathrm{FeCo} / \mathrm{MgO}$ interface. Appl Phys Lett. (2013) 102:152401. doi: 10.1063/1.4802030

26. Bauer U, Yao L, Tan AJ, Agrawal P, Emori S, Tuller HL, et al. Magneto-ionic control of interfacial magnetism. Nat Mater (2015) 14:174-81. doi: $10.1038 /$ nmat4134

27. Koyama T, Obinata A, Hibino Y, Hirohata A, Kuerbanjiang B, Lazarov VK, et al. Dependence of Curie temperature on Pt layer thickness in Co/Pt system. Appl Phys Lett. (2015) 106:132409. doi: 10.1063/1.4916824

28. Arrott A. Criterion for ferromagnetism from observations of magnetic isotherms. Phys Rev. (1957) 108:1394-6: doi: 10.1103/PhysRev.108.1394

29. Arrott A, Noakes JE. Approximate equation of state for nickel near its critical temperature. Phys Rev Lett. (1967) 19:786-9. doi: 10.1103/PhysRevLett.19.786

30. Schneider CM, Bressler P, Schuster P, Kirschner J, de Miguel JJ, Miranda R. Curie temperature of ultrathin films of fcc-cobalt epitaxially grown on 
atomically flat $\mathrm{Cu}(100)$ surfaces. Phys Rev Lett. (1990) 64:1059-62. doi: 10.1103/PhysRevLett.64.1059

31. Shimamura K, Chiba D, Ono S, Fukami S, Ishiwata N, Kawaguchi M, et al. Electrical control of Curie temperature in cobalt using an ionic liquid film. Appl Phys Lett. (2012) 100:122402. doi: 10.1063/1.3695160

32. Takahashi C, Ogura M, Akai H. First-principles calculation of the Curie temperature Slater-Pauling curve. J Phys Condens Matter (2007) 19:365233. doi: 10.1088/0953-8984/19/36/365233

33. Oba M, Nakamura K, Akiyama T, Ito T, Weinert M, Freeman AJ. Electricfield-induced modification of the magnon energy, exchange interaction, and Curie temperature of transition-metal thin films. Phys Rev Lett. (2015) 114:107202. doi: 10.1103/PhysRevLett.114.107202

34. MacDonald AH, Daams JM, Vosko SH, Koelling DD. Influence of relativistic contributions to the effective potential on the electronic structure of Pd and Pt. Phys Rev B (1981) 23:6377-98. doi: 10.1103/PhysRevB.23.6377

35. Sigalas MM, Papaconstantopoulos DA. Calculations of the total energy, electron-phonon interaction, and Stoner parameter for metals. Phys Rev B (1994) 50:7255-61. doi: 10.1103/PhysRevB.50.7255

36. Shinohara T, Sato T, Taniyama T. Surface ferromagnetism of Pd fine particles. Phys Rev Lett. (2003) 91:197201. doi: 10.1103/PhysRevLett.91.197201

37. Sun Y, Burton JD, Tsymbal EY. Electrically driven magnetism on a Pd thin film. Phys Rev B (2010) 81:064413. doi: 10.1103/PhysRevB.81.064413

38. Shimizu S, Takahashi KS, Hatano T, Kawasaki M, Tokura Y, Iwasa Y. Electrically tunable anomalous Hall effect in Pt thin films. Phys Rev Lett. (2013) 111:216803. doi: 10.1103/PhysRevLett.111.216803

39. Obinata A, Hibino Y, Hayakawa D, Koyama T, Miwa K, Ono S, et al. Electricfield control of magnetic moment in Pd. Scientific Rep. (2015) 5:14303. doi: $10.1038 /$ srep 14303

40. Gradmann U, Bergholz R. Magnetization of Pd (111) films by contact with ferromagnetic Ni (111) films. Phys Rev Lett. (1984) 52:771-4. doi: 10.1103/PhysRevLett.52.771

41. den Broeder FJA, Donkersloot HC, Draaisma HJG, de Jonge WJM. Magnetic properties and structure of Pd/Co and Pd/Fe multilayers. J Appl Phys. (1987) 61:4317-9. doi: 10.1063/1.338459
42. Wu R, Li C, Freeman AJ. Structual, electronic and magnetic properties of Co/Pd(111) and Co/Pt(111). J Magn Magn Mater (1991) 99:71-80. doi: 10.1016/0304-8853(91)90048-F

43. Cheng L, Altounian Z, Ryan DH, Ström-Olsen JO, Sutton M, Tun Z. Pd polarization and interfacial moments in Pd-Fe multilayers. Phys Rev B (2004) 69:144403. doi: 10.1103/PhysRevB.69.144403

44. Suzuki M, Muraoka H, Inaba Y, Miyagawa H, Kawamura N, Shimatsu T, et al. Depth profile of spin and orbital magnetic moments in a subnanometer Pt film on Co. Phys Rev B (2005) 72:054430. doi: 10.1103/PhysRevB.72. 054430

45. Hase TPA, Brewer M, Arnalds UB, Ahlberg M, Kapaklis V, Björck M, et al. Proximity effects on dimensionality and magnetic ordering in $\mathrm{Pd} / \mathrm{Fe} / \mathrm{Pd}$ trilayers. Phys Rev B (2014) 90:104403. doi: 10.1103/PhysRevB.90.104403

46. Schellekens AJ, van den Brink A, Franken JH, Swagten HJM, Koopmans B. Electric-field control of domain wall motion in perpendicularly magnetized materials. Nat Comm. (2012) 3:847. doi: 10.1038/ncomms1848

47. Chiba D, Kawaguchi M, Fukami S, Ishiwata N, Shimamura K, Kobayashi $\mathrm{K}$, et al. Electric-field control of magnetic domain-wall velocity in ultrathin cobalt with perpendicular magnetization. Nat Comm. (2012) 3:888. doi: $10.1038 /$ ncomms 1888

48. Bauer U, Emori S, Beach GSD. Electric field control of domain wall propagation in Pt/Co/GdOx films. Appl Phys Lett. (2012) 100:192408. doi: $10.1063 / 1.4712620$

Conflict of Interest Statement: The author declares that the research was conducted in the absence of any commercial or financial relationships that could be construed as a potential conflict of interest.

Copyright (C) 2015 Chiba. This is an open-access article distributed under the terms of the Creative Commons Attribution License (CC BY). The use, distribution or reproduction in other forums is permitted, provided the original author(s) or licensor are credited and that the original publication in this journal is cited, in accordance with accepted academic practice. No use, distribution or reproduction is permitted which does not comply with these terms. 